\title{
Localization of electronic states by fullerene charges in carbon nanotubes
}

\author{
J. González ${ }^{1}$ and F. Guinea ${ }^{2}$ \\ ${ }^{1}$ Instituto de Estructura de la Materia, Consejo Superior de Investigaciones Científicas, Serrano 123, 28006 Madrid, Spain \\ ${ }^{2}$ Instituto de Ciencia de Materiales de Madrid, Consejo Superior de Investigaciones Científicas, Cantoblanco, 28049 Madrid, Spain
}

(Received 5 November 2004; revised manuscript received 27 December 2004; published 31 May 2005)

\begin{abstract}
We study the effects of the electrostatic interaction produced by charged metallofullerenes encapsulated in carbon nanotubes, showing that they are able to modify locally the electronic density of states in the hybrid system. In the cases where the interaction is felt as an attractive potential by the electrons in the nanotube, localized electronic states are formed in the nanotubes around the position of the fullerenes. This produces an effective narrowing of the gap in semiconducting nanotubes over a distance of a few nanometers, in agreement with the spatial modulation of the gap observed in the experiments.
\end{abstract}

DOI: 10.1103/PhysRevB.71.193409

PACS number(s): 73.22.-f, 71.10.Pm

Carbon nanotubes currently have a great potential for technological applications in devices at the nanometer scale. This makes it very important to have precise knowledge of their electronic properties, once the first step of discerning their metallic or insulating behavior has been completed after confirmation of the theoretical analyses ${ }^{1}$ by experimental results. $^{2}$ We have learned from these studies that the lowenergy spectrum of a carbon nanotube may be gapless or not, depending on the helicity of the tubular structure.

Hybrid structures have been recently synthesized by encapsulation of fullerenes in the hollow space of the nanotubes. It has been shown that the insertion of the fullerene molecules in these so-called peapods leads to a significant modification of the electronic spectrum of the carbon nanotubes, opening the possibility to tailor different structures with the desired functionality. In the experiment reported in Ref. 3, chains of $\mathrm{C}_{60}$ molecules have been encapsulated in the interior of semiconducting nanotubes, showing that the measured spectra can be explained from the hybridization of the electronic states in the nanotubes with those in the fullerenes. In Ref. 4, a different kind of peapod has been studied, formed by the encapsulation of sparse metallofullerenes Gd @ $\mathrm{C}_{82}$ in semiconducting nanotubes. In that case, a striking observation has been the spatial modulation of the nanotube band gap, which is narrowed from $0.43 \mathrm{eV}$ down to $0.17 \mathrm{eV}$ at the fullerene positions.

Several electronic-structure calculations have shown that some levels of the fullerene molecular orbitals may lie within the gap of the semiconducting nanotubes. ${ }^{5,6}$ These results have been used to explain the narrowing of the band gap observed experimentally by claiming that, at the fullerene sites, the measured gap should correspond to the distance from the top of the valence band to the lowest unoccupied molecular level within the nanotube gap. This kind of effective reduction of the gap seems to be in agreement with the value measured in the experiments. On the other hand, it has been already pointed out in Ref. 5 that a more detailed study of the states within the gap would be required to account for its continuous modulation along the longitudinal direction of the nanotube.

The distinctive feature of the peapods displaying the modulation of the band gap lies in the charge of the encapsulated metallofullerenes. It is natural to assume that the $\mathrm{Gd}$ element, as in other chemical compounds, will be in an ionic state (the most common oxidation state being $\mathrm{Gd}^{3+}$ ). As shown in Ref. 5, the metallofullerenes give away the electron from the highest occupied molecular orbital in the experimental setup described in Ref. 4. Thus, they acquire a net positive charge and, besides the effect of the empty molecular level left within the gap, it becomes pertinent to take into account the Coulomb interaction between the net charge of the metallofullerenes and the electrons in the nanotube.

In the present paper, we show that the Coulomb interaction produced by the charged metallofullerenes is able to modify locally the electronic density of states of the peapods. In the cases where the net charge $Q_{z}$ gives rise to an attractive potential for the electrons in the nanotube, localized electronic states are formed in the nanotubes around the position of the fullerenes. Their spatial extension as well as their number can be estimated by scaling arguments following from the particular form of the potential. For that purpose, we can rely on a one-dimensional (1D) low-energy model of the semiconducting nanotubes, which will allow the determination of the bound states formed by the Coulomb potential within the gap. The description of the localized states induced by the Coulomb interactions using a basis built up from states near the edges of the valence and conduction band is standard in the study of impurities in semiconductors. The main difference, in the present case, is the reduction of screening effects due to the $1 \mathrm{D}$ geometry. This, in turn, implies stronger interaction effects, which we will estimate through a consistent description of the screening processes using bosonization methods.

We illustrate our 1D low-energy description in the case of the zigzag nanotubes. These have a unit cell with length $3 a$, in terms of the $C$ - $C$ distance $a$. For each transverse array of atoms $n=1,2, \ldots, N$ at a given nanotube section $x$, it is convenient to introduce the Fourier transform of the electron operators $c(x, n)$,

$$
c(x, n) \sim \sum_{p} d_{p}(x) e^{i 2 \pi n p / N},
$$

where the index $p$ labels the different subbands $p$ $=0, \pm 1, \ldots, \pm N / 2$. Within the tight-binding approach, the Hamiltonian for the zigzag nanotube is 


$$
\begin{aligned}
H_{t b}= & -t_{\|} \sum_{p, l} d_{p}^{+}(3 l a) d_{p}(3 l a+a)-t_{\perp} \sum_{p, l} z_{p} d_{p}^{+}(3 l a+a) d_{p}(3 l a \\
& +3 a / 2)-t_{\|} \sum_{p, l} d_{p}^{+}(3 l a+3 a / 2) d_{p}(3 l a+5 a / 2) \\
& -t_{\perp} \sum_{p, l} z_{p}^{*} d_{p}^{+}(3 l a+5 a / 2) d_{p}(3(l+1) a)
\end{aligned}
$$

with $z_{p}=1+\exp (i 2 \pi p / N)$ and $l \in Z$. Henceforth, we will consider that the transfer integrals for the two different bond orientations can be taken approximately equal, $t_{\perp}=t_{\|}=t$. Within this description, each band is obtained from the diagonalization of a one-dimensional chain with two orbitals per unit cell, with gap $2 \Delta_{p}=2 t|1-2 \cos (\pi p / N)|$. This simple one-particle description accounts for the semiconducting character of the zigzag nanotubes when $N$ is not a multiple of 3.

In the semiconducting nanotube, we can write $N$ $=3 N^{\prime} \pm 1$. The subbands closest to the Fermi level have $p$ $\approx N^{\prime}$, and a dispersion typical of a 1D Dirac spinor with Fermi velocity $v_{F}=3 \mathrm{ta} / 2$ and a mass parameter $m=\Delta_{p}$, $\varepsilon(k) \approx \pm \sqrt{v_{F}^{2} k^{2}+m^{2} .}{ }^{7}$ In the following, we assume that the metallofullerene potential has radial symmetry, so that the subband index $p$ remains a good quantum number. Then, the calculation of the perturbed energy levels can be factorized into a set of equivalent problems, one for each subband of the nanotube. For the sake of describing the many-body effects in the interaction with the metallofullerene charge $Q_{z}$, it is most convenient to write the model for the low-energy electronic states in terms of the 1D Dirac spinor field with components $\Psi_{L}$ and $\Psi_{R}$. We will assume that the charge $Q_{z}$ has a finite extension as seen along the longitudinal dimension of the nanotube, being centered at a given position $z$ and spreading over a distance $R$ of the order of the fullerene radius. The Hamiltonian of the interacting theory becomes then for each low-energy subband

$$
\begin{aligned}
H= & \int d x\left(-i v_{F} \Psi_{L}^{+} \partial_{x} \Psi_{L}+i v_{F} \Psi_{R}^{+} \partial_{x} \Psi_{R}+m \Psi_{L}^{+} \Psi_{R}+m \Psi_{R}^{+} \Psi_{L}\right) \\
& +\frac{e^{2}}{2} \int d x d y\left[\rho_{L}(x)+\rho_{R}(x)\right] \frac{1}{|x-y|}\left[\rho_{L}(y)+\rho_{R}(y)\right] \\
& -Q_{z} e^{2} \int d x d y \frac{1}{\sqrt{\pi} R} e^{-(x-z)^{2} / R^{2}} \frac{1}{|x-y|}\left[\rho_{L}(y)+\rho_{R}(y)\right], \quad(3)
\end{aligned}
$$

where $\rho_{L}(x), \rho_{R}(x)$ are the respective electron density operators for the two spinor components. ${ }^{8}$

The effect of the metallofullerene charge in the electronic spectrum can be understood by applying the semiclassical approximation to the electron wave functions. In this approach, we assume that the only significant effect of the electron-electron interaction within the nanotube is to induce some charge distribution screening the net charge of the metallofullerene. The screening is not complete, as it is produced by a system with a gap at the Fermi level, and it can be encoded at a later stage in the form of a suitable dielectric function. At this point, we concentrate therefore on the resolution of the eigenvalue problem,

$$
\begin{gathered}
{\left[-i v_{F} \partial_{x}+V(x-z)\right] \Psi_{L}+m \Psi_{R}=\varepsilon \Psi_{L},} \\
{\left[i v_{F} \partial_{x}+V(x-z)\right] \Psi_{R}+m \Psi_{L}=\varepsilon \Psi_{R},}
\end{gathered}
$$

where $V(x-z)$ stands for the potential created by the charge $Q_{z}$ centered at $z$.

The system (4) and (5) can be reduced to an equation for any of the two components of the spinor. In the semiclassical approximation, the $\Psi_{R}$ component reads, for instance,

$$
\begin{aligned}
\Psi_{R}(x) \approx & \frac{e^{ \pm(1 / 2) \int^{x} d x \partial_{x} V(x-z) / \sqrt{[\varepsilon-V(x-z)]^{2}-m^{2}}}}{\left\{[\varepsilon-V(x-z)]^{2}-m^{2}\right\}^{1 / 4}} \\
& \times e^{ \pm i \int^{x} d x\left(1 / v_{F}\right) \sqrt{[\varepsilon-V(x-z)]^{2}-m^{2}}} .
\end{aligned}
$$

The turning points are given by the roots of the equation $\left[\varepsilon-V\left(r_{0}\right)\right]^{2}=m^{2}$. When such points exist, the semiclassical approximation gives rise to the quantization condition

$$
\int_{-r_{0}}^{r_{0}} d x \sqrt{[\varepsilon-V(x)]^{2}-m^{2}}=v_{F} \pi\left(n+\frac{1}{2}\right) .
$$

When the potential $V(x)$ has the long-distance behavior $V(x) \sim-1 /|x|$, it can be shown from Eq. (7) that there is an infinite series of bound states with energy below and approaching the value of $m$. If we define $m-\varepsilon=\epsilon$, the quantization condition translates into

$$
\int_{-r_{0}}^{r_{0}} d x \sqrt{[-V(x)-\epsilon][2 m-V(x)-\epsilon]}=v_{F} \pi\left(n+\frac{1}{2}\right) .
$$

As the parameter $\epsilon$ goes to zero, we observe that the integral on the left-hand side of the equation scales as $1 / \sqrt{\epsilon}$, reflecting the divergent range of integration. Thus, for large values of $n$, we find the quantization rule

$$
m-\varepsilon_{n} \sim \frac{1}{(n+1 / 2)^{2}} .
$$

The above arguments convey that the Coulomb potential of the metallofullerene charge has to induce localized electronic states within the nanotube gap. We show next that this conclusion holds after incorporating the screening of the potential by the electronic charge in the nanotube. Our continuum model allows actually an exact treatment of such an effect by means of bosonization methods. ${ }^{9}$ Following these, the electron density for a given subband near the Fermi level can be mapped into a boson field $\Phi(x)$ according to the relation $(1 / 2 \pi) \partial \Phi(x)=\rho_{L}(x)+\rho_{R}(x)$. In terms of the boson fields, the Hamiltonian (3) can be expressed in the form

$$
\begin{aligned}
H= & \frac{1}{2} \int d x\left(4 \pi v_{F} \Pi^{2}(x)+\frac{v_{F}}{4 \pi}\left[\partial_{x} \Phi(x)\right]^{2}+\frac{2 m}{\pi \alpha} \cos [\Phi(x)]\right) \\
& +\frac{e^{2}}{8 \pi^{2}} \int d x d y \partial_{x} \Phi(x) \frac{1}{|x-y|} \partial_{y} \Phi(y) \\
& -\frac{Q_{z} e^{2}}{2 \pi} \int d x d y \frac{1}{\sqrt{\pi} R} e^{-(x-z)^{2} / R^{2}} \frac{1}{|x-y|} \partial_{y} \Phi(y)
\end{aligned}
$$

where $\Pi(x)$ is the momentum conjugate of the field $\Phi(x)$ and 


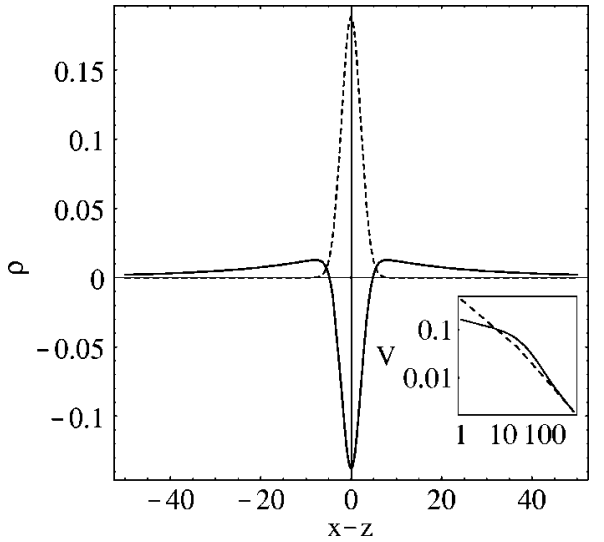

FIG. 1. Plot of the model fullerene charge distribution for $Q_{z}$ $=1$ (dashed line) and of the screening charge distribution induced in the carbon nanotube (full line). The distance $x$ is measured with respect to the center of the charge $Q_{z}$ at point $z$, in units of the $C$ - $C$ distance in the nanotube. Inset: $\log -\log$ plot of the bare Coulomb potential (dashed line) and of the dressed potential obtained after considering the screening charge in the nanotube (full line).

$\alpha$ is a short-distance cutoff needed to regulate the product of fields at the same point.

From the representation (10), we observe that the presence of the charge $Q_{z}$ induces a nonvanishing average value of the field $\partial_{x} \Phi(x)$. As we are interested in low-energy screening properties, we can expand the $\cos [\Phi(x)]$ dependence to deal with a Hamiltonian quadratic in the boson fields. The screening charge distribution can be obtained then by shifting the field $\Phi(x)$ by a suitable function, $\Phi(x)-f(x)$ $=\widetilde{\Phi}(x)$, so that the redefined density has a vanishing average, $\left\langle\partial_{x} \widetilde{\Phi}(x)\right\rangle=0$. The approach allows a straightforward generalization to the case of $N_{f}$ different electron flavors, accounting for spin and subband degeneracies. Thus, the expression for the screening electron density induced in the nanotube is in general

$$
\begin{aligned}
\rho_{s}(x)= & \frac{1}{2 \pi}\langle\partial \Phi(x)\rangle=Q_{z} \int \frac{d k}{2 \pi} \cos [k(x-z)] e^{-R^{2} k^{2} / 4} \\
& \times \frac{\frac{2 N_{f} e^{2}}{\pi v_{F}} k^{2} \log \left(1+k_{0} / k\right)}{k^{2}+\frac{4 m}{\alpha v_{F}}+\frac{2 N_{f} e^{2}}{\pi v_{F}} k^{2} \log \left(1+k_{0} / k\right)},
\end{aligned}
$$

where the logarithmic dependences come from the representation of the bare Coulomb potential in momentum space. ${ }^{10}$ The shape of the screening charge distribution has been plotted in Fig. 1 for $m / t=0.1$ and $R=3 a$, taking $N_{f}=4$ and $e^{2} / v_{F} \approx 2.7$. We observe that in general there is only a partial screening of the charge $Q_{z}$ around its center at $z$, due to the absence of gapless excitations in the semiconducting nanotube. The results are not qualitatively changed if other subbands with higher gaps are also included.

The dressed Coulomb potential $V_{d}(x-z)$ felt in the nanotube is created by the sum of the background charge $Q_{z} e$ centered at point $z$ and the screening charge $-e \rho_{s}(x)$. Thus we have

$$
\begin{aligned}
V_{d}(x-z)= & -Q_{z} e^{2} \int \frac{d k}{2 \pi} \cos [k(x-z)] e^{-R^{2} k^{2} / 4} \\
& \times \frac{\left(k^{2}+\frac{4 m}{\alpha v_{F}}\right) 2 \log \left(1+k_{0} / k\right)}{k^{2}+\frac{4 m}{\alpha v_{F}}+\frac{2 N_{f} e^{2}}{\pi v_{F}} k^{2} \log \left(1+k_{0} / k\right)} .
\end{aligned}
$$

We observe that the presence of the gap (accounted for by the $m$ parameter) precludes an effective reduction of the Coulomb interaction at small momenta. The dressed potential $V_{d}(x)$ (for the same choice of parameters made above) has been represented in the inset of Fig. 1, where it is also compared with the bare Coulomb potential.

From the above analysis, it is clear that the dressed interaction keeps the long-range character of the Coulomb potential. Thus, in the more complete picture accounting for the screening effects within the nanotube, the result of having localized states due to the presence of the metallofullerene charge remains unmodified. The actual number of them depends on the value $Q_{z}$ of the net metallofullerene charge, as well as on the length of the carbon nanotube. The form of the spectrum within the gap can be obtained for a particular system by diagonalizing the lattice Hamiltonian made of the tight-binding term (2) and the interaction term with the dressed potential

$$
H_{\text {int }}=\sum_{p, i} V_{d}\left(x_{i}-z\right) d_{p}^{+}\left(x_{i}\right) d_{p}\left(x_{i}\right),
$$

where $x_{i}$ label the different sections of atoms in the nanotube, in the same fashion as in Eq. (2).

The results of diagonalizing the Hamiltonian $H_{t b}+H_{\text {int }}$ for a semiconducting $(17,0)$ nanotube have been represented in Fig. 2. The level of the deepest state within the gap depends on the net charge $Q_{z}$, but there is always an accumulation of states near the conduction-band edge. Using the continuum approximation, a state within the gap, at energy $-m \leqslant \varepsilon<m$, is localized within a length $l \approx v_{F} / \sqrt{m^{2}-\varepsilon^{2}}$. This estimate agrees well with the numerical calculations based on the tight-binding model. Hence, the localization length of the deepest levels within the gap is comparable to the diameter of the nanotube. Note that each subband will give rise to a set of localized states within its gap.

The existence of localized states within the gap of the semiconducting nanotube has a significant impact on the density of states of the system. This depends on the value of the net charge $Q_{z}$ and, for each particular experimental setup, on the amount of screening due to external charges. For the sake of drawing a correspondence with the experiments reported in Ref. 4, we have adopted a phenomenological approach by taking an effective value $Q_{z} \approx 0.5$, so that the deepest localized state is near the center of the nanotube gap. The local variation of the density of states can be assessed in terms of the spatial extension of the states which are deep within the gap. These have been obtained from the diagonalization of the lattice Hamiltonian $H_{t b}+H_{\text {int }}$ for a $(17,0)$ nano- 


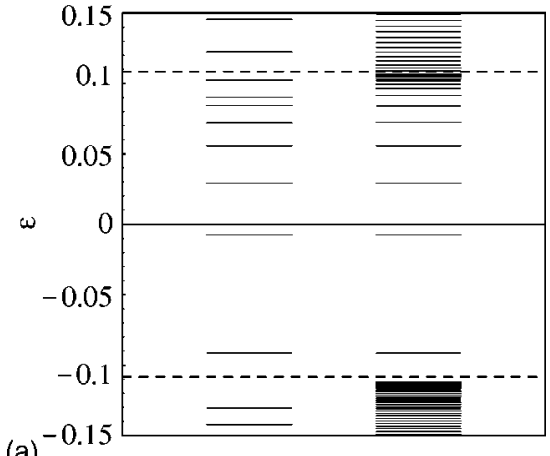

(a)

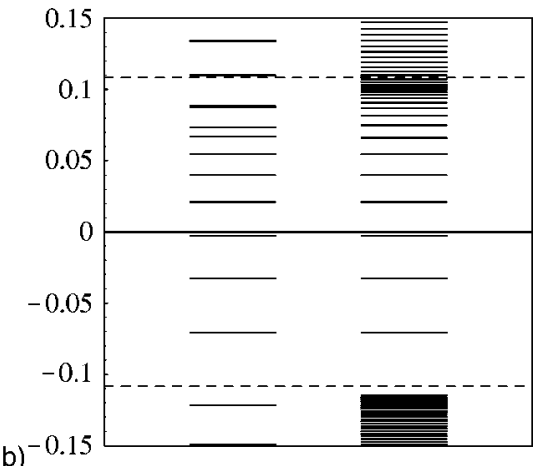

FIG. 2. Diagram of the low-energy levels obtained from the diagonalization of the lattice Hamiltonian for $Q_{z}=0.5$ (a) and $Q_{z}=1.0$ (b). The spectra are given in each case for a couple of nanotube segments with lengths $L=300 a$ and $1500 a$. The energies are in units of the transfer integral $t$. The dashed lines mark the border of the gap in the absence of the metallofullerene charge.

tube, and they are represented in Fig. 3. We observe that their shape is consistent with the local variation of the gap measured in Ref. 4 around the position of each fullerene cluster. In particular, we notice that the deepest states extend over a distance of a few nanometers, in agreement with the spatial modulation of the gap observed in the experiment.

Our results show that the Coulomb interaction between the fullerene clusters and the nanotube has to be taken into account to achieve a consistent explanation of the spatial modulation of the gap in the peapods. In this respect, our approach is complementary to those studies that have fo-
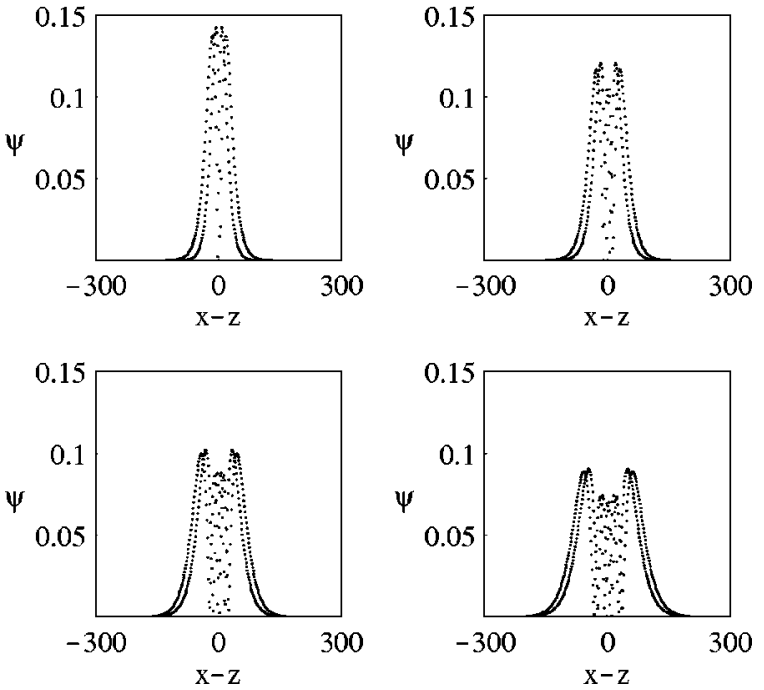

FIG. 3. Plot of the absolute value of the wave functions for the deepest levels within the gap in the top diagram of Fig. 2, given (from left to right and from top to bottom) in order of proximity to the midpoint of the gap. The distance $x$ is measured with respect to the center of the charge $Q_{z}$ at point $z$, in units of the $C$ - $C$ distance in the nanotube. It is noted that the points in each plot form different smooth envelopes, corresponding to different inequivalent positions in the unit cell of the nanotube.

cused on the hybridization of the molecular orbitals of the fullerenes with the nanotube states. The present analysis is consistent with the fact that the narrowing of the gap has been measured in the peapods with metallofullerenes, while no gap reduction has been observed in the samples with encapsulated $\mathrm{C}_{60}$ molecules. Our investigation points to the possibility of having control of the gap reduction in the peapods, by varying the net metallofullerene charge $Q_{z}$. This effect should be confronted in suitably prepared samples, for the sake of making progress in the local band-gap engineering of nanotube devices.

The financial support of the Ministerio de Educación y Ciencia (Spain) through Grants No. MAT2002-0495-C02-01 (for F.G.) and No. BFM2003-05317 (for J.G.) is gratefully acknowledged.
${ }^{1}$ R. Saito, M. Fujita, G. Dresselhaus, and M. S. Dresselhaus, Appl. Phys. Lett. 60, 2204 (1992); J. W. Mintmire, B. I. Dunlap, and C. T. White, Phys. Rev. Lett. 68, 631 (1992); N. Hamada, S. Sawada, and A. Oshiyama, ibid. 68, 1579 (1992).

${ }^{2}$ J. W. G. Wildöer, L. C. Venema, A. G. Rinzler, R. E. Smalley, and C. Dekker, Nature (London) 391, 59 (1998); T. W. Odom, J.-L. Huang, P. Kim, and C. M. Lieber, ibid. 391, 62 (1998).

${ }^{3}$ D. J. Hornbaker, S.-J. Kahng, S. Misra, B. W. Smith, A. T. Johnson, E. J. Mele, D. E. Luzzi, and A. Yazdani, Science 295, 828 (2002).

${ }^{4}$ J. Lee, H. Kim, S.-J. Kahng, G. Kim, Y.-W. Son, J. Ihm, H. Kato, Z. W. Wang, T. Okazaki, H. Shinohara, and Y. Kuk, Nature (London) 415, 1005 (2002).

${ }^{5}$ Y. Cho, S. Han, G. Kim, H. Lee, and J. Ihm, Phys. Rev. Lett. 90,
106402 (2003)

${ }^{6}$ M. Otani, S. Okada, and A. Oshiyama, Phys. Rev. B 68, 125424 (2003).

${ }^{7}$ C. L. Kane and E. J. Mele, Phys. Rev. Lett. 78, 1932 (1997).

${ }^{8}$ We are neglecting here the backscattering interactions, which play a subdominant role in nanotubes of typical radius, as shown by R. Egger and A. O. Gogolin, Phys. Rev. Lett. 79, 5082 (1997), and C. Kane, L. Balents, and M. P. A. Fisher, ibid. 79, 5086 (1997).

${ }^{9}$ See, for instance, A. O. Gogolin, A. A. Nersesyan, and A. M. Tsvelik, Bosonization and Strongly Correlated Systems (Cambridge Univ. Press, Cambridge, UK, 1998).

${ }^{10}$ D. W. Wang, A. J. Millis, and S. Das Sarma, Phys. Rev. B 64, 193307 (2001). 